\title{
Ocorrência de Pb-Zn-Cd (Ag) em Cabeceiras do Lajeado, Rio Grande do Sul - Brasil
}

\author{
João AngeloToniolo¹ (joao.toniolo@cprm.gov.br) \\ Giovani Nunes Parisi ${ }^{1}$ (giovani.parisi@cprm.gov.br) \\ Luiz Gustavo Rodrigues Pinto² (luiz.pinto@cprm.gov.br) \\ Andrea Sander.1.(andrea.sander@cprm.gov.br) \\ Jorge Henrique Laux¹(jorge.laux@cprm.gov.br)
}

${ }^{1}$ CPRM - Serviço Geológico do Brasil, Gerência de Geologia e Recursos Minerais, Porto Alegre ${ }^{2}$ CPRM - Serviço Geológico do Brasil, Divisão de Sensoriamento Remoto e Geofísica, São Paulo

\begin{abstract}
A new Pb-Zn-Cd (Ag) occurrence, named Cabeceiras do Lajeado, was recently discovered by CPRM-Geological Survey of Brazil in the juvenile São Gabriel terrane, Sul-Rio-Grandense Shield, following airborne magnetic and radiometric anomalies. The mineralization is composed of sphalerite, galena and pyrite as the main sulfide phases and is hosted by hornblende gneiss with medium- to fine-grained hornblende needles, with grain size less than $5 \mathrm{~mm}$. The hornblende gneiss shows mineralogical banding composed of quartz, amphibole and sulfides, and of amphibole, quartz, feldspars and/or very fine-grained cordierite. Euhedral to globular pink garnet and chloritized biotite are also present. $\mathrm{Pb}$ and $\mathrm{Zn}(>10,000 \mathrm{ppm})$, Se $(>100 \mathrm{ppm})$ and $\mathrm{Cd}(>2,000 \mathrm{ppm})$ contents exceeded the detection limits of the ICP-MS technique, whereas Ag grades $60.5 \mathrm{ppm}$.
\end{abstract}

Keywords: lead, zinc, Sul-Rio-Grandense Shield, base metals, mineral resources.

Palavras chave: chumbo, zinco, escudo Sul-Rio-Grandense, metais de base, recursos minerais.

\section{INTRODUÇÃO}

Neste trabalho é apresentada a ocorrência inédita de $\mathrm{Pb}-\mathrm{Zn}-\mathrm{Cd}(\mathrm{Ag})$ na região denominada Cabeceiras do Lajeado, identificada pela CPRM - Serviço Geológico do Brasil durante a execução do projeto Agrominerais do Rio Grande do Sul, a partir de verificação em campo de anomalias aerogeofísicas caracterizadas por forte assinatura magnética e baixa radiação gamaespectrométrica. A ocorrência tem como coordenadas UTM 773946 S / 6618307 E e está situada no Escudo Sul-Rio-Grandense (Figura 1).

\section{CONTEXTO GEOLÓGICO}

A área da ocorrência insere-se na porção meridional do Estado do Rio Grande do Sul, no segmento sul da Província Mantiqueira, e está inserida no Terreno São Gabriel, de idade neoproterozoica. O Terreno São Gabriel consiste em uma associação de rochas metavulcanossedimentares e plutônicas com assinatura isotópica juvenil, tidas como formadas em ambiente de arco magmático e associadas a fragmentos de ofiolitos. 


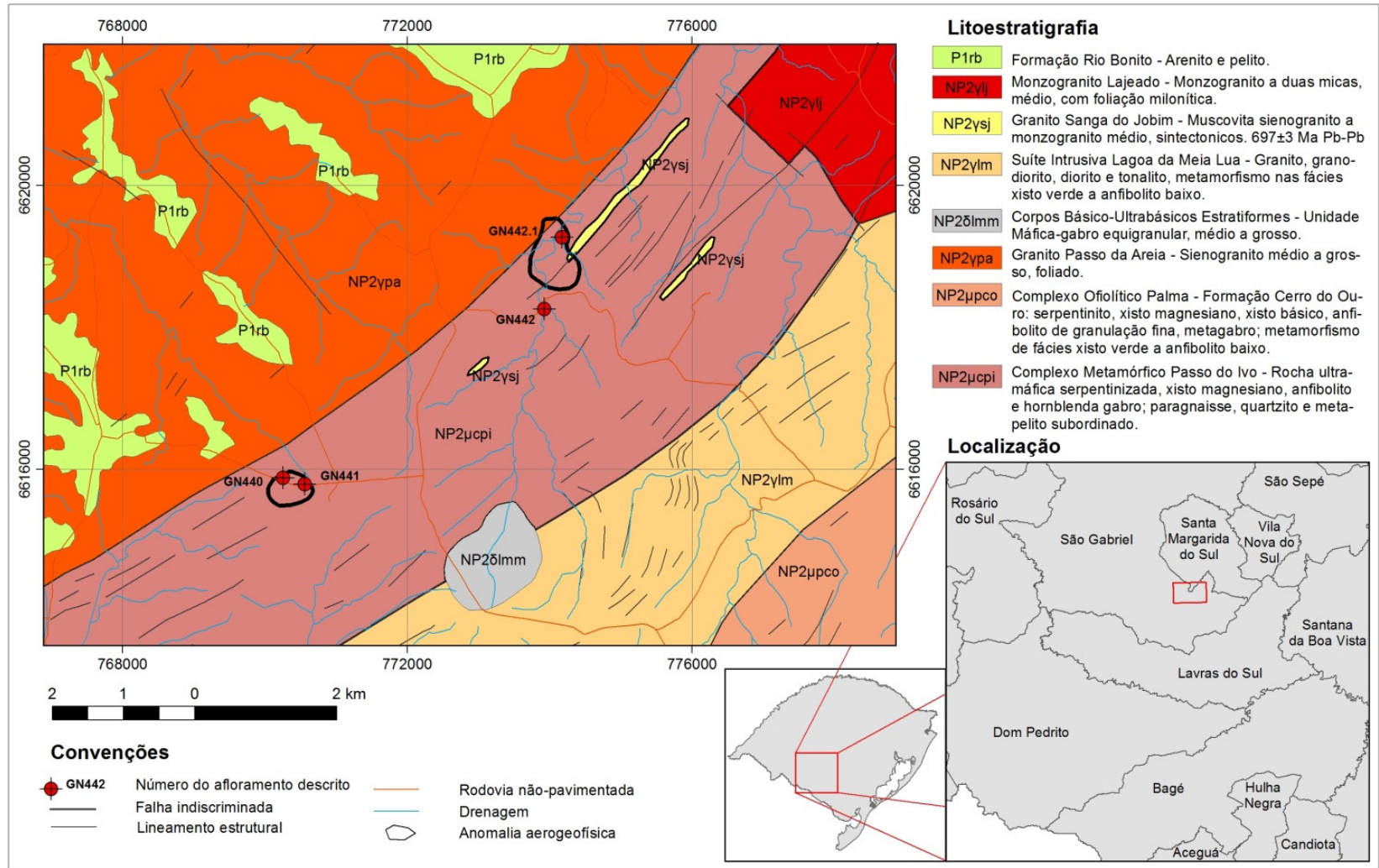

Figura 1 - Localização das anomalias geofísicas e dos pontos amostrados. Geologia a partir de Laux et al. (2012).

\section{GEOLOGIA LOCAL}

A ocorrência mineral de $\mathrm{Pb}-\mathrm{Zn}-\mathrm{Cd}(\mathrm{Ag})$ em Cabeceiras do Lajeado está inserida no Complexo Metamórfico Passo do Ivo (OLIVEIRA, 1982), parte integrante do Terreno São Gabriel, que é constituído por rochas ultramáficas serpentinizadas, xistos magnesianos, anfibolitos, hornblenda gabros, paragnaisses, quartzitos e metapelitos.

Dois alvos principais foram checados na Folha Lagoa da Meia Lua (SH-21-Z-B-VI), sendo um em sua porção centro-nordeste e outro em sua porção sudoeste (Figura 1).

No primeiro alvo, denominado afloramento GN-442, foi identificado hornblenda gnaisse sulfetado que retornou expressivos teores de $\mathrm{Pb}, \mathrm{Zn}, \mathrm{Cd}$ e Ag.

No segundo alvo geofísico, onde ocorrem os afloramentos denominados $\mathrm{GN}-440$ e GN-441, foram descritos filonito, constituído por quartzo e mica, e uma rocha ultramáfica serpentinizada, de cor preta, magnética, isótropa, de grão médio a grosso.

Intrusões sintectônicas de muscovita sienogranito a monzogranito completam a associação de rochas que ocorrem como cristas alongadas na direção $\mathrm{N} 70^{\circ} \mathrm{E}$.

\section{GEOFÍSICA}

A geofísica tem se mostrado uma ferramenta importante na indicação de alvos prospectivos, tendo tido papel fundamental no desenvolvimento do projeto Agrominerais do Rio Grande do Sul, da CPRM (relatório final em elaboração).

Diversas ocorrências minerais foram identificadas a partir do processamento dos dados aerogeofísicos no referido projeto, com destaque para fosfato em carbonatitos, formações ferríferas e esta ocorrência de $\mathrm{Pb}-\mathrm{Zn}-\mathrm{Cd}(\mathrm{Ag})$. Outros alvos, ainda sem avaliação de campo, indicam potencial elevado para novas descobertas minerais.

Os dois alvos avaliados em campo foram selecionados por meio da correlação e integração da primeira derivada vertical e da amplitude do sinal analítico do campo magnético anômalo com dados gamaespectrométricos de composição ternária RGB de $\mathrm{K}$, eTh e eU, a partir de dados do Projeto Aerogeofísico Escudo Rio Grande do Sul (CPRM, 2010).

A Figura 2 corresponde à imagem do sinal analítico da região estudada, submetida a uma modificação na escala de cores normal para realçar as duas anomalias estudadas. Sobrepostas a esta imagem foram plotados os lineamentos magnetométricos interpretados a partir da primeira derivada vertical do campo anômalo, o que evidenciou a relação entre as anomalias magnéticas e as rochas ultramáficas, além de um forte controle desses corpos por lineamentos de direção NE-SW e NW-SE (Figura 2). 


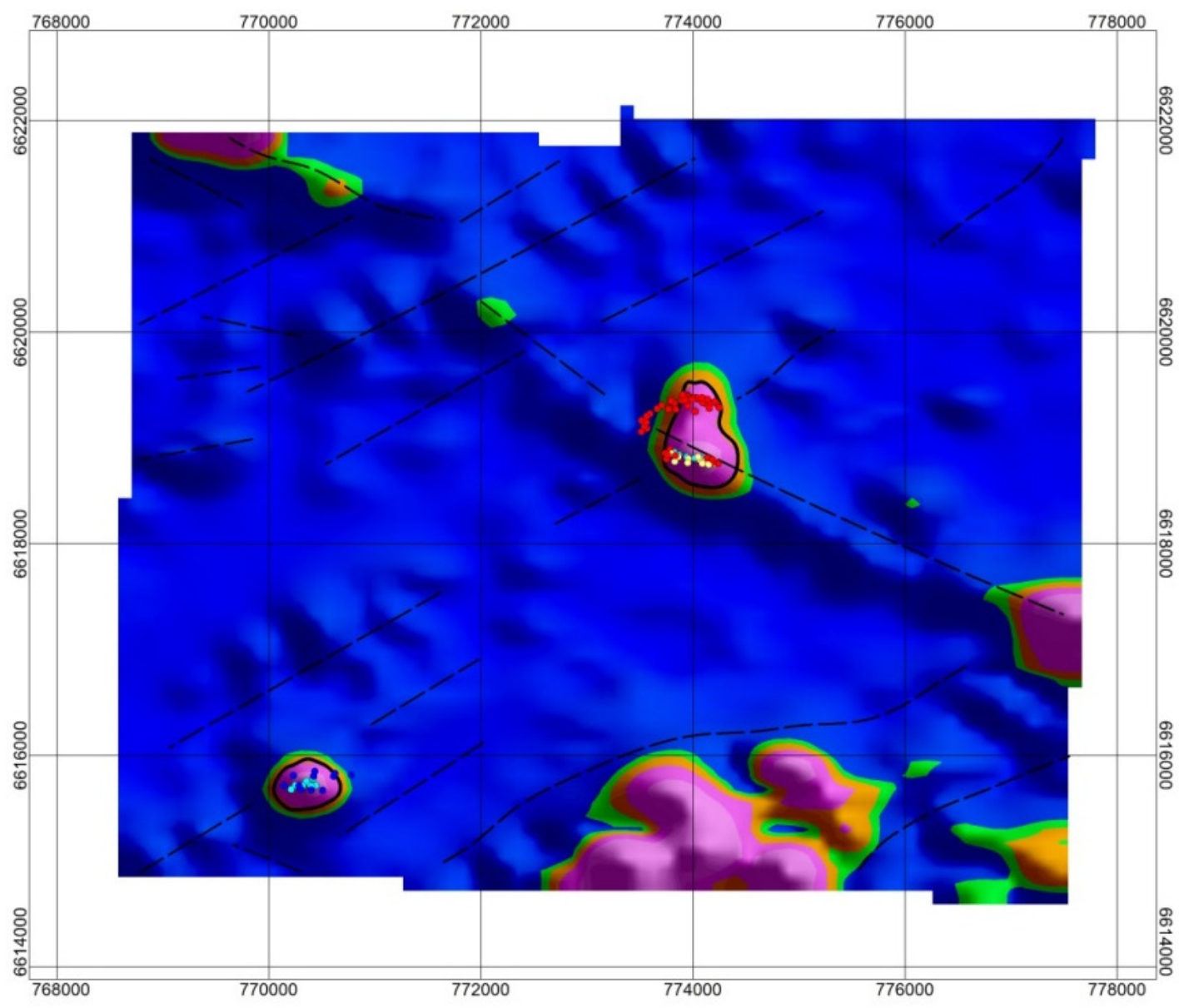

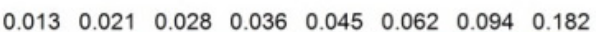

Figura 2 - Mapa da amplitude do sinal analítico com localização das anomalias geofísicas e dos afloramentos descritos. As linhas tracejadas em preto representam os lineamentos magnetométricos interpretados do mapa de primeira derivada vertical do campo magnético anômalo. O círculo em preto representa as duas anomalias avaliadas em campo, destacando-se que a anomalia centro-nordeste, onde ocorre a mineralização de Cabeceiras do Lajeado, se encontra na intersecção de lineamentos magnetométricos NE e NW.

Além disso, foi estimada a profundidade das fontes anômalas, a partir do estudo de interpretação semi-quantitativo com o algoritmo da deconvolução de Euler, com índice estrutural 2, indicando que o corpo causador da anomalia de sudoeste é raso, enquanto o corpo causador da anomalia centro-nordeste não aflora, o que justificaria a não identificação de rochas magnéticas aflorantes a ela associadas.

A Figura 3 apresenta a composição ternária RGB dos elementos $K$, eTh e eU com sobreposição dos lineamentos magnetométricos. Dois domínios gamaespectrométricos principais ocorrem na área, limitados exatamente pela estrutura NE-SW que controla os corpos ultramáficos, sendo esta uma importante indicação para a definição de alvos prospectivos. O domínio a noroeste do lineamento apresenta elevados teores dos elementos $\mathrm{K}$, $\boldsymbol{e} \mathrm{Th}$ e $\boldsymbol{e} \mathrm{U}$, enquanto a sudeste os valores são mais baixos nos três elementos.

Os dois alvos avaliados em campo são representados por valores gamaespectro- métricos baixos, representando a natureza das rochas encaixantes.

Na verificação de campo foram empregados equipamentos para medidas pontuais da susceptibilidade magnética das rochas com kappameter e da radioatividade (canais $e \mathrm{U}, \boldsymbol{e} \mathrm{Th}$ e K) com o gamaespectrômetro.

\section{DESCRIÇÃO DA OCORRÊNCIA}

A ocorrência de Cabeceiras do Lajeado foi descrita em um único afloramento, com cerca de 20 metros de comprimento e espessura visível de 1 metro, no barranco de uma estrada secundária (GN-442), a cerca de um quilômetro da borda da parte principal da anomalia magnética associada ao alvo da porção centro-nordeste da área. Trata-se de um hornblenda gnaisse sulfetado, bastante denso, de coloração cinza escura a esverdeada, não magnética, com foliação de atitude $\mathrm{N} 60^{\circ} \mathrm{E}$, vertical (Figuras $1,4$ e $5 A)$. 

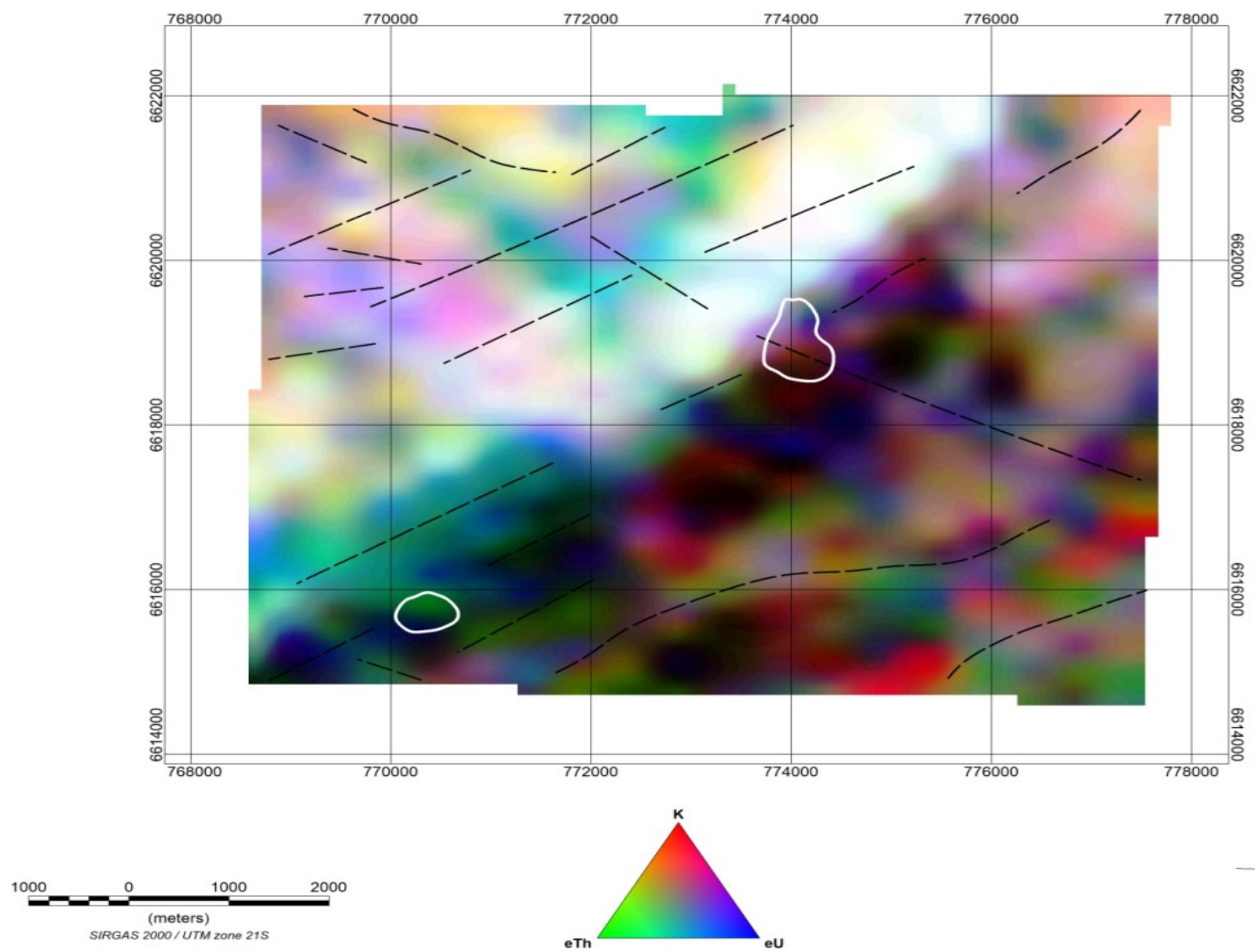

Figura 3 - Mapa da composição ternária RGB da região de estudo. As linhas tracejadas em preto indicam os lineamentos magnetométricos interpretados do mapa de primeira derivada vertical do campo anômalo e os círculos em branco representam as anomalias em estudo.

Sobre a anomalia magnética propriamente dita (ponto GN-442.1 da Figura 1) foram encontrados apenas raros blocos pequenos e soltos de filonito, quartzo branco e granitoide, que não justificaram a anomalia magnética delimitada, sendo a fonte do magnetismo ainda desconhecida.

\section{PETROGRAFIA}

A rocha hospedeira da mineralização corresponde, petrograficamente, a um hornblenda gnaisse bandado com alternância de cores em tons preto, verde e branco (Figura $5 A$ ). Possui textura nematoblástica definida pela orientação de agulhas de anfibólio de grão médio a fino, com tamanho dos grãos inferior a $5 \mathrm{~mm}$ (Figura 5B). O bandamento é marcado pela alternância de níveis ricos em quartzo, no anfibólio hornblenda, esfalerita (15\%), galena $(5 \%)$ e pirita $(5 \%)$ e níveis de hornblenda, quartzo e feldspatos (?), não se descartando a possibilidade de ocorrer cordierita muito fina associada (Figuras 6 e 7). Ocorrem também grãos de granada rosada, euédricos a globulares, pós-cinemáticos, além de algum quartzo intersticial, estirado na direção da foliação, e de biotita cloritizada.

\section{ANÁLISE QUÍMICA}

A amostra GN-442 foi analisada por ICP-MS para óxidos maiores e mais 53 elementos. A análise dos elementos $\mathrm{Pb}(>1 \%)$, $\mathrm{Zn}(>1 \%)$, Se (>100 ppm) e Cd (>2000 ppm) superou os limites de detecção do método utilizado (Tabela 1, valores destacados em vermelho). Cabe também ressaltar os altos valores de Ag (60,5 ppm) e anômalos em Au (53,5 ppb).

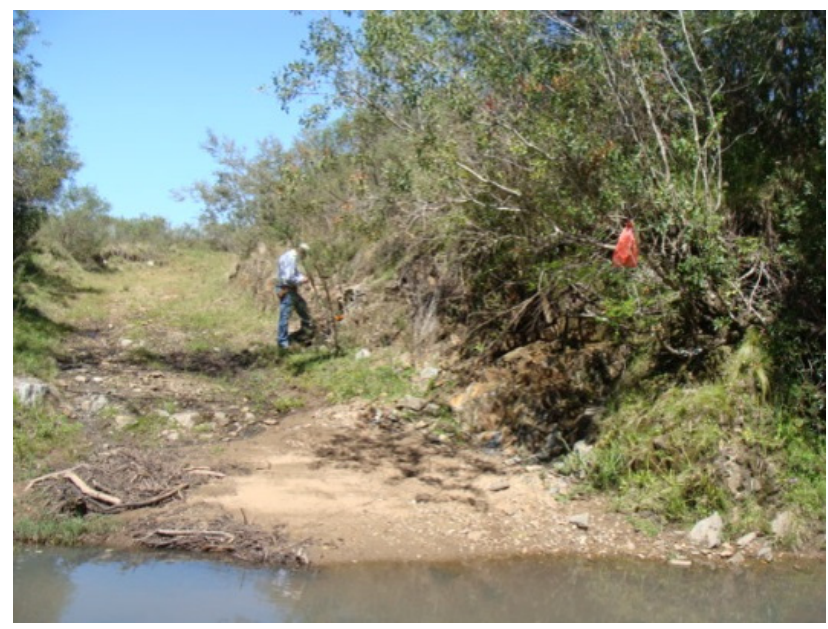

Figura 4 - Afloramento GN-442. 

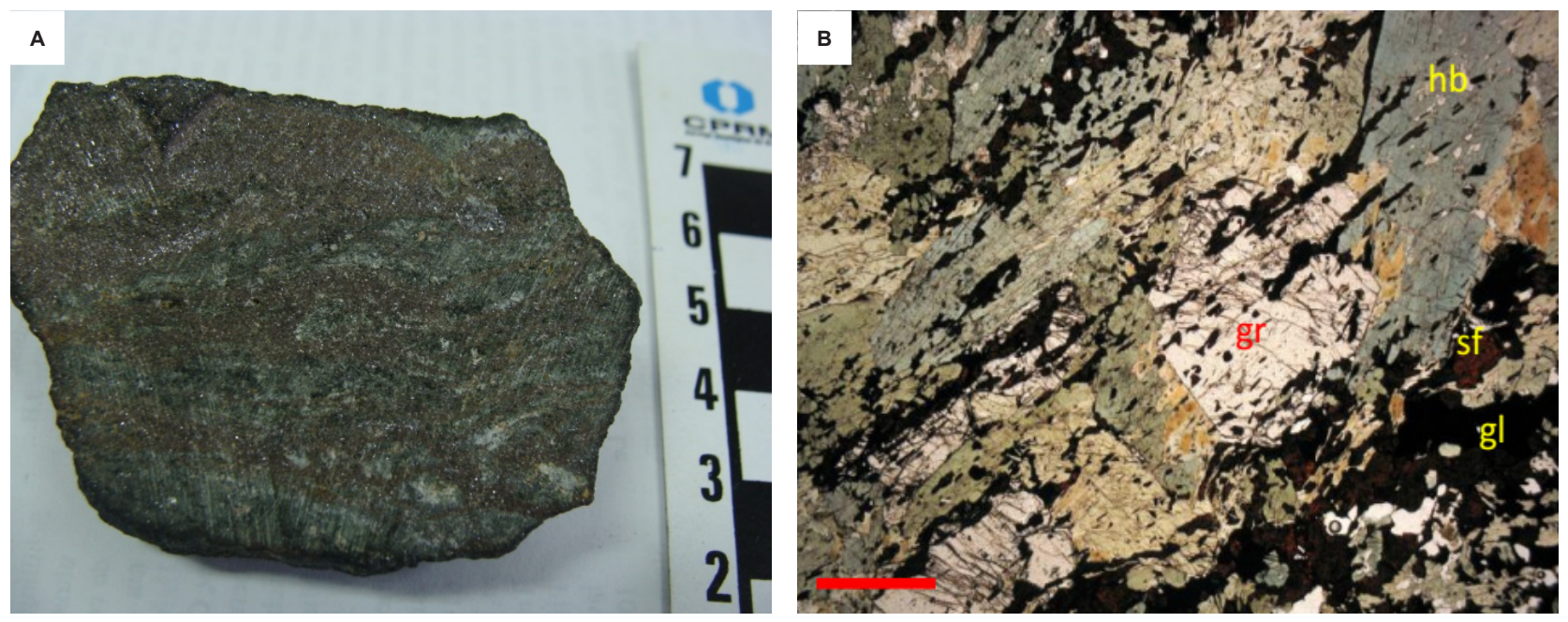

Figura 5 - (A) Hornblenda gnaisse (GN-442) com bandamento marcado pela alternância de níveis ricos em silicatos (com predomínio de quartzo e hornblenda) e níveis mais ricos em sulfetos. (B) - Fotomicrografia do hornblenda gnaisse GN-442 mostrando a textura nematoblástica, com hornblenda (hb) orientada, granada (gr), galena (gl) e esfalerita (sf). Luz polarizada. Escala gráfica de $1 \mathrm{~mm}$.
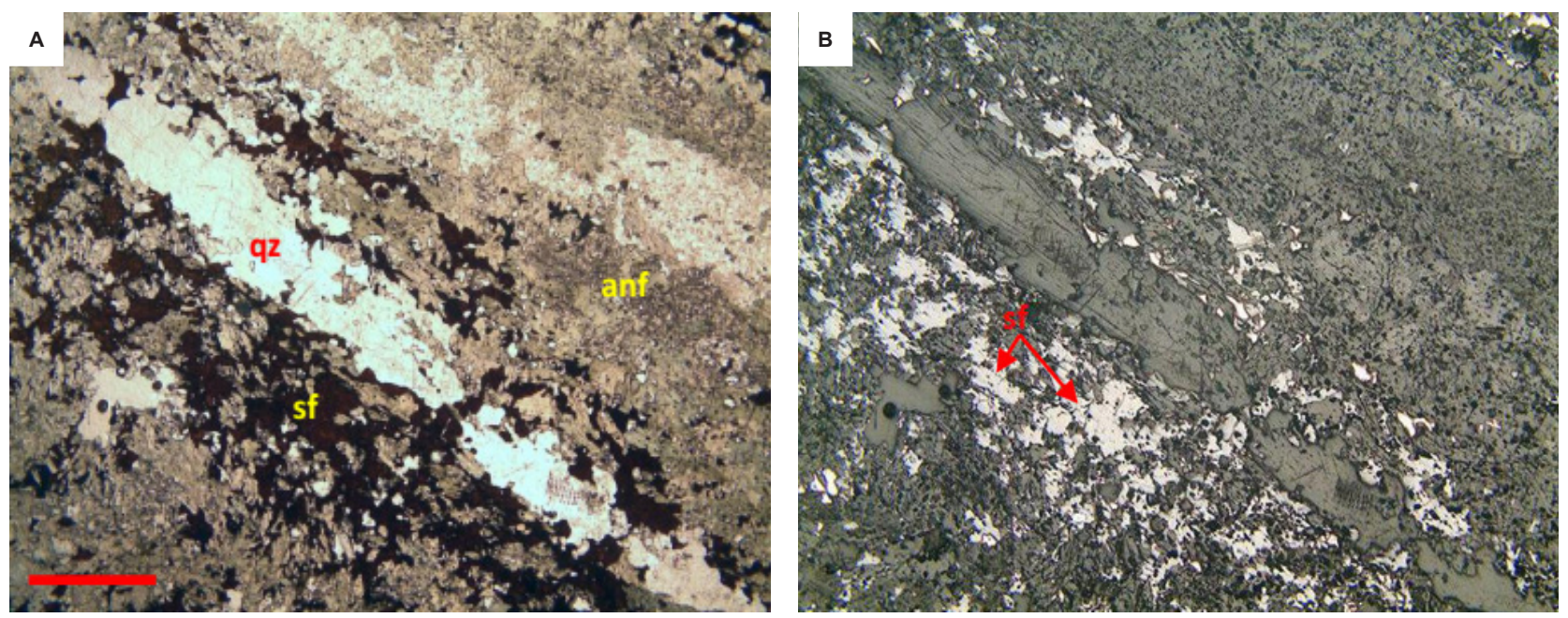

Figura 6 - (A) Hornblenda gnaisse GN-442, com detalhe do bandamento caracterizado pela alternância de níveis claros com anfibólio (anf) e quartzo (qz) e níveis escuros, avermelhados, ricos de esfalerita (sf), em luz plano polarizada com escala gráfica de $1 \mathrm{~mm}$. (B) Mesmo campo de A em luz refletida e mesma escala gráfica, com destaque para a esfalerita (sf).
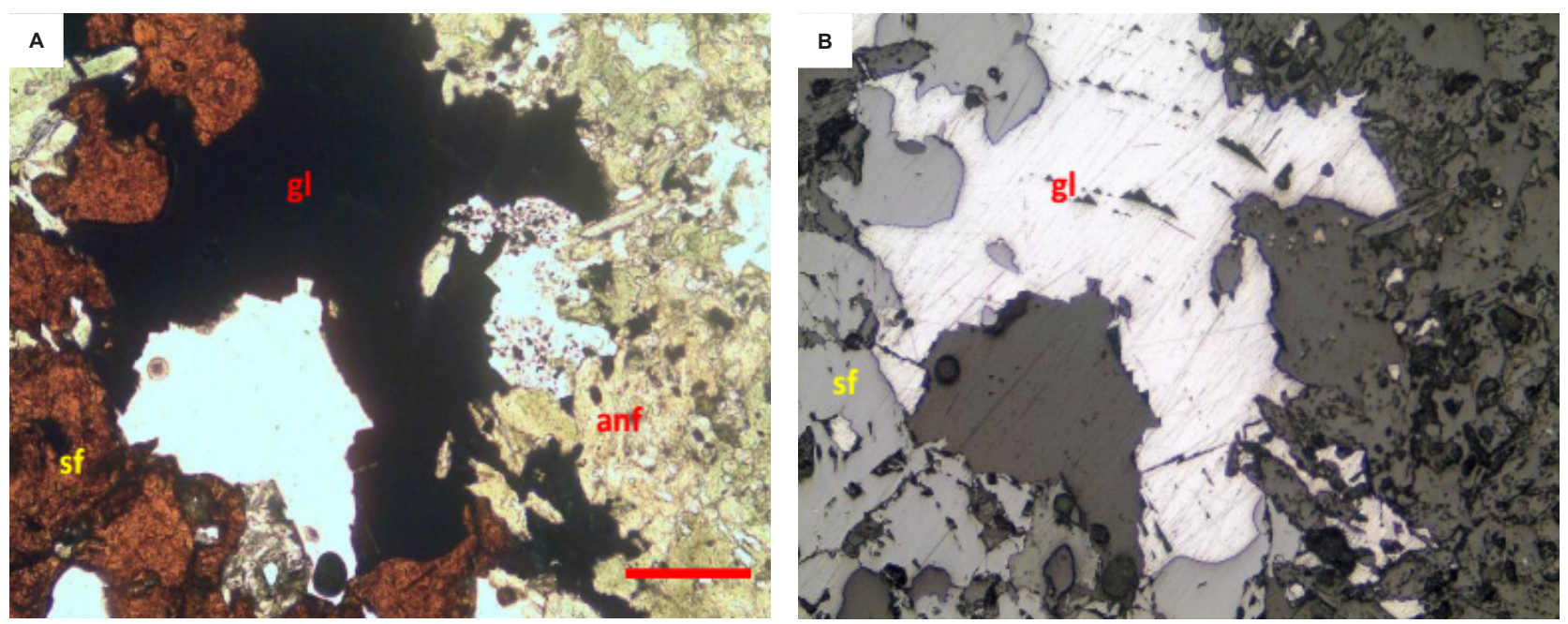

Figura 7 - (A) Hornblenda gnaisse do ponto GN-442, com detalhe da esfalerita (sf) de cor castanho avermelhada em contato com galena (gl) e anfibólios (anf). Luz plano polarizada e escala gráfica de $0,5 \mathrm{~mm}$. (B) Mesmo campo de A em luz refletida com mesma escala gráfica, com destaque para esfalerita (sf) e galena ( $g l$ ) 
Tabela 1 - Resultado analítico da amostra GN-442.

\begin{tabular}{|c|c|c|c|c|c|c|c|c|c|c|c|c|c|c|}
\hline $\begin{array}{c}\mathrm{Al}_{2} \mathrm{O}_{3} \\
\%\end{array}$ & $\begin{array}{c}\mathrm{CaO} \\
\%\end{array}$ & $\begin{array}{c}\mathrm{Cr}_{2} \mathrm{O}_{3} \\
\%\end{array}$ & $\begin{array}{c}\mathrm{Fe}_{2} \mathrm{O}_{3} \\
\%\end{array}$ & $\begin{array}{c}\mathrm{K}_{2} \mathrm{O} \\
\%\end{array}$ & $\begin{array}{c}\text { MgO } \\
\%\end{array}$ & $\begin{array}{c}\text { MnO } \\
\%\end{array}$ & $\begin{array}{c}\mathrm{Na}_{2} \mathrm{O} \\
\%\end{array}$ & $\begin{array}{c}\mathbf{P}_{2} \mathbf{O}_{5} \\
\%\end{array}$ & $\begin{array}{c}\mathrm{SiO}_{2} \\
\%\end{array}$ & $\begin{array}{c}\mathrm{TiO}_{2} \\
\%\end{array}$ & $\begin{array}{c}\text { LOI } \\
\%\end{array}$ & $\begin{array}{c}\text { TOT/C } \\
\%\end{array}$ & $\begin{array}{c}\text { TOT/S } \\
\%\end{array}$ & $\begin{array}{c}\text { Sum } \\
\%\end{array}$ \\
\hline 3,72 & 3,74 & 0,023 & 9,91 & 0,21 & 3,94 & 0,71 & 0,53 & 0,04 & 23,3 & 0,2 & 4,3 & $<0,02$ & 15,91 & 50,63 \\
\hline $\begin{array}{c}\text { Ba } \\
\text { ppm }\end{array}$ & $\begin{array}{c}\text { Be } \\
\text { ppm }\end{array}$ & $\begin{array}{c}\mathrm{Ce} \\
\mathrm{ppm}\end{array}$ & $\begin{array}{c}\text { Co } \\
\mathrm{ppm}\end{array}$ & $\begin{array}{c}\text { Cs } \\
\mathrm{ppm}\end{array}$ & $\begin{array}{c}\text { Dy } \\
\text { ppm }\end{array}$ & $\begin{array}{c}\text { Er } \\
\text { ppm }\end{array}$ & $\begin{array}{c}\text { Eu } \\
\mathrm{ppm}\end{array}$ & $\begin{array}{c}\text { Ga } \\
\mathrm{ppm}\end{array}$ & $\begin{array}{c}\text { Gd } \\
\mathrm{ppm}\end{array}$ & $\begin{array}{c}\text { Hf } \\
\text { ppm }\end{array}$ & $\begin{array}{c}\text { Ho } \\
\text { ppm }\end{array}$ & $\begin{array}{c}\text { La } \\
\text { ppm }\end{array}$ & $\begin{array}{c}\text { Lu } \\
\text { ppm }\end{array}$ & $\begin{array}{c}\mathbf{N b} \\
\mathrm{ppm}\end{array}$ \\
\hline 243 & 3 & 8,8 & 89,6 & 0,4 & 1,12 & 0,66 & 0,44 & 6,2 & 1,37 & 1,4 & 0,26 & 6,4 & 0,11 & 2,6 \\
\hline $\begin{array}{c}\text { Nd } \\
\mathrm{ppm}\end{array}$ & $\begin{array}{c}\mathbf{N i} \\
\mathrm{ppm}\end{array}$ & $\begin{array}{c}\text { Pr } \\
\text { ppm }\end{array}$ & $\begin{array}{c}\mathbf{R b} \\
\mathrm{ppm}\end{array}$ & $\begin{array}{c}\text { Sc } \\
\mathrm{ppm}\end{array}$ & $\begin{array}{c}\text { Sm } \\
\mathrm{ppm}\end{array}$ & $\begin{array}{c}\text { Sn } \\
\mathrm{ppm}\end{array}$ & $\begin{array}{c}\mathrm{Sr} \\
\mathrm{ppm}\end{array}$ & $\begin{array}{c}\text { Ta } \\
\text { ppm }\end{array}$ & $\begin{array}{c}\text { Tb } \\
\mathrm{ppm}\end{array}$ & $\begin{array}{c}\text { Th } \\
\text { ppm }\end{array}$ & $\begin{array}{c}\text { Tm } \\
\text { ppm }\end{array}$ & $\begin{array}{c}\mathbf{U} \\
\mathrm{ppm}\end{array}$ & $\begin{array}{c}\mathbf{V} \\
\mathrm{ppm}\end{array}$ & $\begin{array}{c}\mathbf{W} \\
\mathrm{ppm}\end{array}$ \\
\hline 5,7 & 119 & 1,23 & 6,6 & 5 & 1,19 & 74 & 79,2 & 0,2 & 0,18 & 1,3 & 0,13 & 0,2 & 76 & $<0,5$ \\
\hline $\begin{array}{c}\mathbf{Y} \\
\text { ppm }\end{array}$ & $\begin{array}{c}\text { Yb } \\
\text { ppm }\end{array}$ & $\begin{array}{c}\mathbf{Z r} \\
\mathrm{ppm}\end{array}$ & $\begin{array}{c}\text { Ag } \\
\text { ppm }\end{array}$ & $\begin{array}{c}\text { As } \\
\mathrm{ppm}\end{array}$ & $\begin{array}{c}\mathrm{Au} \\
\mathrm{ppm}\end{array}$ & $\begin{array}{c}\text { Bi } \\
\text { ppm }\end{array}$ & $\begin{array}{c}\text { Cd } \\
\text { ppm }\end{array}$ & $\begin{array}{c}\mathrm{Cu} \\
\mathrm{ppm}\end{array}$ & $\begin{array}{c}\mathrm{Hg} \\
\mathrm{ppm}\end{array}$ & $\begin{array}{c}\text { Mo } \\
\text { ppm }\end{array}$ & $\begin{array}{c}\mathbf{N i} \\
\text { ppm }\end{array}$ & $\begin{array}{c}\mathrm{Pb} \\
\mathrm{ppm}\end{array}$ & $\begin{array}{c}\text { Sb } \\
\mathrm{ppm}\end{array}$ & $\begin{array}{c}\text { Se } \\
\mathrm{ppm}\end{array}$ \\
\hline 8,2 & 0,85 & 41,5 & 60,5 & $<0,5$ & 53,5 & 1,2 & $>2000$ & 25,0 & 2,03 & 2,6 & 41,9 & $>10000$ & 41,6 & $>100$ \\
\hline $\begin{array}{c}\text { TI } \\
\text { ppm }\end{array}$ & $\begin{array}{c}\text { Zn } \\
\text { ppm }\end{array}$ & & & & & & & & & & & & & \\
\hline 0,8 & $>10000$ & & & & & & & & & & & & & \\
\hline
\end{tabular}

\section{CONSIDERAÇÕES FINAIS}

As verificações de anomalias aerogeofísicas pelo projeto Agrominerais do Rio Grande do Sul identificou, até o momento, os carbonatitos Três Estradas e Joca Tavares, além de diversos outros indícios de áreas prospectivas no Escudo Sul-Riograndense. Dentre essas áreas encontra-se uma nova ocorrência de $\mathrm{Pb}-\mathrm{Zn}-\mathrm{Cd}(\mathrm{Ag})$ no Terreno São Gabriel, denominada de Cabeceiras do Lajeado.

Os macrocontroles estruturais que permitiram identificar essas recentes descobertas da CPRM no Escudo Sul-Rio-Grandense são anomalias aeromagnetométricas e gamaespectrométricas situadas principalmente no cruzamento de lineamentos NE e NW.

Nessa mesma região, a jusante da ocorrência aqui descrita, levantamentos geoquímicos em drenagens indicaram a presença de anomalias de ouro em concentrados de bateia (TONIOLO; KIRCHNER, 2001) e teores anômalos de $\mathrm{Pb}, \mathrm{Zn}$ e $\mathrm{Ag}$ em amostras de sedimento de corrente (93 ppm Pb, 54 ppm $\mathrm{Zn}$ e $49 \mathrm{ppm} \mathrm{Ag}$ ) em drenagem localizada a montante da ocorrência (LAUX et al., 2012), demonstrando a possibilidade de existir novos alvos prospectivos nas proximidades.

A descoberta da ocorrência de $\mathrm{Pb}-\mathrm{Zn}$ - Cd (Ag) de Cabeceiras do Lajeado abre a perspectiva para o desenvolvimento de trabalhos prospectivos pela iniciativa privada, sendo esta uma importante contribuição da CPRM-Serviço Geológico do Brasil para o desenvolvimento do setor mineral do país.
O trabalho realizado mostra ainda a importância do uso da geofísica como ferramenta de prospecção mineral no Escudo Sul-Rio-Grandense.

\section{REFERÊNCIAS}

CPRM - SERVIÇO GEOLÓGICO DO BRASIL. Projeto Aerogeofísico Escudo do Rio Grande do Sul: relatório final do levantamento e processamento dos dados magnetométricos e gamaespectométricos. Rio de Janeiro: Lasa Prospecções, 2010. 27 v. Programa Geologia do Brasil - PGB.

LAUX, Jorge Henrique; BONGIOLO, Everton Marques; KLEIN, Carla; IGLESIAS, Carlos Moacyr da Fontoura. Geologia e Recursos Minerais da Folha Lagoa da Meia Lua, SH.21-Z-B-VI, Estado do Rio Grande do Sul, escala 1:100.000. Porto Alegre: CPRM, 2012. (Programa Geologia do Brasil).

OLIVEIRA, Marisa Terezinha Garcia de. Petrologia do Maciço Máfico-Ultramáfico Passo do Ivo, São Gabriel, Rio Grande do Sul. Acta Geológica Leopoldensia, São Leopoldo, v.6, n.11, p. 131-218, 1982.

TONIOLO, João Angelo; KIRCHNER, Carlos Alberto. Resultados da prospecção para o ouro na área RS01, Lavras do Sul-Caçapava do Sul,subárea Passo do Ivo Rio Grande do Sul. Porto Alegre: CPRM, 2001. 1 v. 2 mapas Escala 1:75.000. (Informe de Recursos Minerais. Série Ouro, Informes Gerais, no 14). Programa Nacional de Prospecção de Ouro, PNPO. 


\section{INFORME TÉCNICO N 5}

Brasília, maio 2016

ISSN: 2248-2242

\section{Publicação on-line seriada}

CPRM - Serviço Geológico do Brasil.

Disponível em: www.cprm.gov.br

\section{CPRM - Serviço Geológico do Brasil}

SBN - Quadra 02 - Bloco H, Ed. Central Brasília, $1^{\circ}$ andar Brasília - DF - Brasil

CEP: 70040-904

Telefone:(61) 2108-8400

www.cprm.gov.br

contatos: seus@cprm.gov.br

evandro.klein@cprm.gov.br
Diretor de Geologia e Recursos Minerais

Roberto Ventura Santos

Corpo Editorial

Evandro Luiz Klein (Editor)

João Henrique Larizzatti,

Luiz Gustavo Rodrigues Pinto,

Edilton José dos Santos

\section{Revisores}

Marco Tulio N. Carvalho,

Marcus F. N. Chiarini

Normalização Bibliográfica

Nelma Fabrícia da P. Ribeiro Botelho

\section{Editoração Eletrônica}

Marcelo Henrique Borges Leão

Nelma Fabrícia da P. Ribeiro Botelho 